\title{
A RARE CASE ON CHRONIC BUDD- CHIARI SYNDROME
}

Balaji Arumugam, Elangkumaran Manoharan, Saranya Nagalingam

1. Assistant Professor. Department of Community Medicine, ACS Medical College. Chennai.

2. Tutor. Department of Community Medicine, ACS Medical College. Chennai.

3. M.B.B.S Student. Department of Community Medicine, ACS Medical College. Chennai.

\section{CORRESPONDING AUTHOR:}

Dr. A. Balaji,

Assistant Professor,

Department of Community Medicine,

A.C.S. Medical College and Hospital,

Velappanchavadi, Chennai - 600077.

E-mail: dr.a.balaji@gmail.com

ABSTRACT: Budd-Chiari syndrome is the clinical picture caused by occlusion of the hepatic veins. It presents with the abdominal pain, ascites and hepatomegaly. The syndrome can be fulminant, acute, chronic, or asymptomatic. One such middle aged female patient came to the clinic with history of chronic jaundice, ascites, and hyper pigmentation of both legs. After complete physical examination and investigations, the reports revealed membranous web causing obstruction of inferior vena cava resulting in venous stasis of the Lower limbs and in turn the hepatic veins has resulted in esophageal varices with early liver cell necrosis and jaundice.

KEY WORDS - IVC obstruction, membranous web, esophageal varices, non healing ulcers, jaundice, ascites, Collaterals, hepatic veins, Budd Chiari Syndrome.

INTRODUCTION: Budd-Chiari syndrome is a heterogeneous group of disorders characterized by hepatic venous outflow obstruction at the level of the hepatic venules, the large hepatic veins, the inferior vena cava, or the right atrium ${ }^{1}$. Obstruction is usually caused by a thrombus, but may result from extrinsic compression (tumour, abscess, cysts), membranous webs within the inferior vena cava (IVC) ${ }^{2}$. The syndrome present with spectrum of signs and symptoms and the diagnosis can be made non-invasively when imaging shows venous obstruction and/or collaterals. So imaging plays an important role in diagnosing and confirming the condition.

HISTORY: A 27 year old married graduate female named Mrs. Jayasree, presented with swelling and hyper pigmentation of both legs for the past one year and abdominal pain and distension, jaundice and for 5 months duration. She was apparently normal before one year, the swelling of her both legs started gradually and later on, the swelling continued with itching, pain on walking and she noted the non-healing ulcers in her both lower limbs, for which she was undergoing treatment with a dermatologist. For jaundice and abdominal pain she has taken some native treatment. She is married since 2 years, as she had two early abortions she was also consulting a gynaecologist. She came to the clinic for that non healing ulcers and hyper pigmentation which was bothering her more than the jaundice or abdominal pain.

EXAMINATION: On general examination, she was moderately nourished, pallor and icterus was present. On inspection, both anterior and posterior aspects of her legs were discoloured, with healed scars, cracks and ulcers. On Inspection of the abdomen, dilated veins were seen over the 
flanks, and on palpation tender hepatomegaly and splenomegaly were noted. Liver was enlarged two fingers below right costal margin and was tender, abdominal distension was noted. Then on percussion shifting dullness was observed, which was suggestive of free fluid (ascites). Mild tenderness was observed throughout the abdomen during palpation and percussion. She was referred for complete Haematological investigations, USG abdomen, followed by CT abdomen. The patient was also sent for UGI scopy to rule out esophageal varices as she was having ascites.

INVESTIGATIONS: Ultrasonography of the abdomen confirmed the presence of Moderate Ascites and Hepato-Splenomegaly. Decreased Platelet count ( 0.43 lakhs per cubic millimetre of blood) was detected during Haematological investigations. Increased values of liver function tests were also observed which indicated an on-going process of Hepatic Necrosis. Upper Gastro-intestinal endoscopy revealed the presence of esophageal varices. CT whole abdomen indicated Coarctation of Inferior Vena Cava due to a membranous web with Multiple Intra and Extra Hepatic Collateral veins. With these above findings, suggests that this patient is a Rare Case of Budd- Chiari Syndrome.

DISCUSSION: BCS is a complex disease with a wide spectrum of etiologies and presentations. Chronic case of Budd-Chiari syndrome presents with Abnormal Liver Function tests and dilated tortuous veins in the flanks along with pedal edema, oesophageal varices and HepatoSplenomegaly. The Classical trend to diagnose Budd-Chiari Syndrome is abdominal pain, intractable ascites and Hepato-Splenomegaly. In this case, along with Classical trend the patient also presented with the complications such as IVC obstruction due to membranous web resulting in lower limb venous stasis, esophageal varices, Hepato-Splenomegaly with signs of liver failure. Membranous web occlusion of the IVC is much more common in the Asian population than in western countries ${ }^{3}$. In addition to these findings, she was ordered for Anti nuclear antibodies for ruling out systemic cause which was also found to be positive.

A multidisciplinary approach to diagnosis and treatment is advantageous in patients with BCS. Medical therapy consists of treatment of Underlying Disease, Anticoagulation and Symptom control. Emerging technologies have offered new Minimally Invasive treatment modalities such as Percutaneous Catheter-directed Thrombolysis, Angioplasty, Stenting and TIPS. While surgical shunting continues to play a major role in the treatment of patients with BCS, its place in treatment strategies is unclear ${ }^{4}$.

\section{REFERENCES:}

1. Ludwig J, Hashimoto E, McGill DB, van Heerden JA. Classification of hepatic venous outflow obstruction: ambiguous terminology of the Budd-Chiari syndrome. Mayo Clin Proc 1990;65:51-5.

2. Langlet $P$, Valla D. Is surgical portosystemic shunt the treatment of choice in Budd-Chiari syndrome? Acta Gastroenterol Belg 2002; 65: 155-60.

3. Chung RT, Iafrate AJ, Amrein PC, Sahani DV, Misdraji J. Case records of the Massachusetts general hospital. Case 15- 2006. A 46-year-old woman with sudden onset of abdominal distention. N Engl J Med 2006; 354: 2166-75.

4. John D. Horton, Francisco L. San Miguel and Jorge A. Ortiz, Budd Chiari syndrome: illustrated review of current management. Liver International ISSN 1478-3223 


\section{CT ABDOMEN FILM SHOWING MEMBRANOUS WEB AND HEPATIC VEIN COLLATERALS}

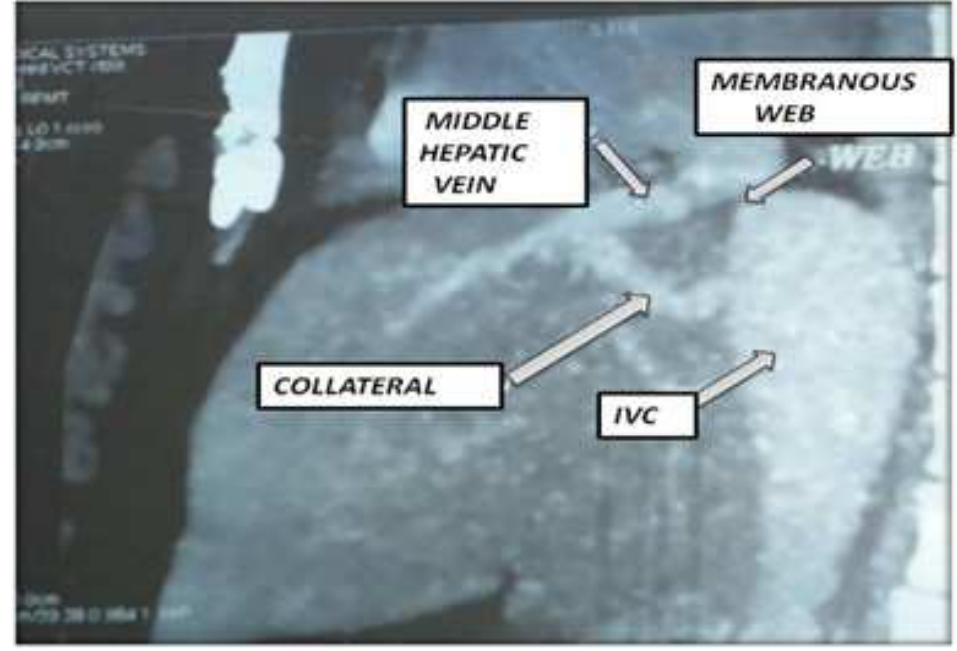

ULTRASONOGRAM - SHOWING SITE OF OBSTRUCTION AND HEPATIC VEIN COLLATERALS

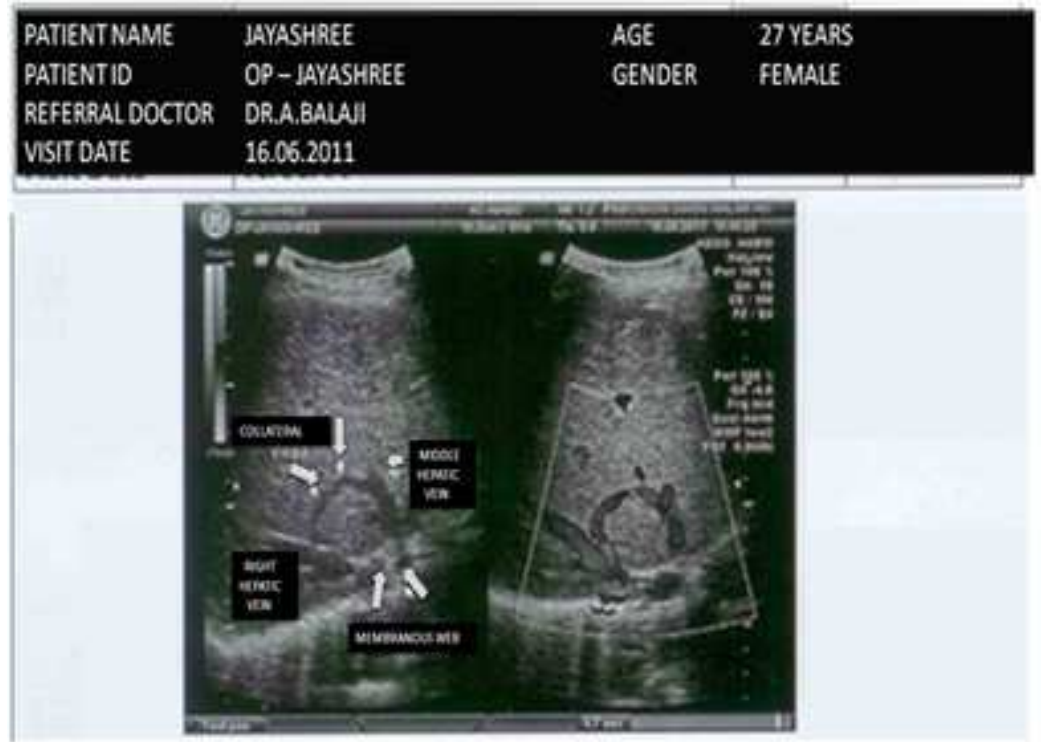

